\title{
Voltron: A Hybrid System For Answer Validation Based On Lexical And Distance Features
}

\author{
Ivan Zamanov ${ }^{1}$, Nelly Hateva ${ }^{1}$, Marina Kraeva ${ }^{1}$, Ivana Yovcheva ${ }^{1}$, \\ Ivelina Nikolova ${ }^{2}$, Galia Angelova ${ }^{2}$ \\ ${ }^{1}$ FMI, Sofia University, Sofia, Bulgaria \\ ${ }^{2}$ IICT, Bulgarian Academy of Sciences, Sofia, Bulgaria \\ ivo.zamanov@gmail.com, nelly.hateva@gmail.com, mvkraeva@gmail.com, \\ ivana.yovcheva@gmail.com, iva@lml.bas.bg, galia@lml.bas.bg
}

\begin{abstract}
The purpose of this paper is to describe our submission to the SemEval-2015 Task 3 on Answer Selection in Community Question Answering. We participated in subtask A, where the systems had to classify community answers for a given question as definitely relevant, potentially useful, or irrelevant. For every question-answer pair in the training data we extract a vector with a variety of features. These vectors are then fed to a MaxEnt classifier for training. Given a question and an answer the trained classifier outputs class probabilities for each of the three desired categories. The one with the highest probability is chosen. Our system scores better than the average score in subtask A of Task 3.
\end{abstract}

\section{Introduction}

Nowadays, text analysis and semantic similarity are subject to a lot of research and experiments due to the growth of social media influence, the increasing usage of forums for finding a solution of common known problems and the Web upgrowth. As beginners in the computational linguistics field, we were very interested in dealing with these topics and have found Answer Validation as a good start. Our team chose to focus on subtask A of Task 3 in the SemEval-2015 workshop, namely Answer selection in community question answering data. In order to achieve good results, we combined most of the techniques familiar to us. We process the data as question-answer pairs. The framework GATE (Cunningham et al., 2002) was used for the preprocess- ing in the system because it offers convenient natural language processing pipelines and has an API allowing for system integration. For classification we used the Maximum Entropy classifier provided by MALLET (McCallum and Kachites, 2002). We use a combination of surface, morphological, syntactic, and contextual features as well as distance metrics between the question and answer. Distance metrics are based on word2vec (Mikolov et al., 2013a) and DKPro Similarity (Bär, et al.), (de Castilho, 2014).

\section{Related work}

Several recent systems were created and used for similar analysis. Although their applications have some differences from the system described in this paper, we consider them relevant because they deal with semantic similarity.

(Başkaya, 2014) uses Vector Space Models which have some similarity to our usage of word2vec centroid metrics with the difference that we do not organize the whole text according to the structure of the result matrix, as the VSMs do. The cosine similarity is common for both systems. The big difference is that we use only the input words while in his system the words' likely synonyms according to a language model are also used. We believe this contributes to the consistently higher scores of his system.

Another work of (Vilarin̂o et al., 2014) also uses n-grams, cosine similarity and that is a common feature with our system. Some differing features are Jaccard coefficient, Latent Semantic Analysis, Pointwise Mutual Information. Their results are very close to ours.

Most of the works dealing with semantic similar- 
ity use n-grams, metadata features and stop words as we do. Our scores are not among the highest in subtask A of Task 3, but they come close to and substantially differ from the average score in this field of works.

\section{Resources}

The datasets we use to train our system are provided from the SemEval-2015 organizers. The datasets consist of 2600 training and 300 development questions including 16,541 training and 1,645 development comments.

Also for the extraction of some features we use pretrained word and phrase vectors. They are trained on part of Google News dataset (about 100 billion words). The model contains 300-dimensional vectors for 3 million words and phrases.

\section{Method}

The task at hand is to measure how appropriate and/or informative a comment is with respect to a question. Our approach is to measure the relatedness of a comment to the question or, in other words, to measure if a question-comment pair is consistent. Therefore we attempt to classify each pair as Good, Potential or Bad.

The main characteristic of a good comment is that it is related to the corresponding question. Also, we assume that when answering a question, people tend to use the same words with which the question was asked because that would make it easier for the question author to understand. Therefore, similar wording and especially similar phrases would be an indication of a more informative comment.

\subsection{Features}

We will call tokens that are not punctuation or stop words meaningful, as they carry some information regardless of exactly how a sentence is formulated.

\subsubsection{Lexical Features}

For every meaningful token, we extract its stem, lemma and orthography.

\subsubsection{N-gram Features}

Bigrams and trigrams of tokens (even nonmeaningful ones) are also extracted since this should capture similar phrases used in the questioncomment pair. We assume that n-grams of higher order could contribute as well, however we believe $n=2$ and $n=3$ would carry the most information and $n \geq 4$ would impact training time adversely.

\subsubsection{Bad-answer-specific Features}

Bad comments often include a lot of punctuation, more than one question in the answer, questions, followed by their obvious answer (when the expression or its synonyms could be directly found in the answer), more than two repeating letters next to each other (i.e. exclamations such as "ahaa"), greetings, chat abbreviations, more than one uppercase word, a lot of emoticons, exclamations and other very meaningless words. Emphasizing such tokens helps to distinguish bad comments specifically.

\subsubsection{Structural Features}

We include the comment's length in meaningful tokens, length in sentences and each sentence's length as features, since longer comments should include more information. Since named entities, such as locations and organizations etc. would be especially indicative of the topic similarity between question and comment, we give them greater weight by again including named entities, recognized by GATE's built-in NER tools.

\subsubsection{TF Vector Space Features}

Another attempt to capture similar terms in the question and comment is to convert each entry to a local term-frequency vector and compute the cosine similarity between the vectors for the question and comment rounded to 0.1 precision. Similar wording, regardless of term occurrence frequency, should lead to a higher cosine similarity. We use DKPro's implementation of cosine similarity to achieve this (Bär, et al.). The term "local" refers to the fact that $\mathrm{TF}$ vectors of distinct entries are not related, that is, the vector space is specific to a question-comment pair.

\subsubsection{Word2vec Semantic Similarity}

A good answer, however, does not necessarily use the exact same words. Therefore we need a way to capture the general "topic" of a question. We opted for the word2vec word vectors, proposed by (Mikolov et al., 2013a), (Mikolov et al., 
2013b), (Mikolov et al., 2013c). The general idea of word $2 \mathrm{vec}$ is to represent each word as a real vector that captures the contexts of word occurrences in a corpus. For a given question-comment pair, we extract word2vec vectors from a pre-trained set for all tokens for which one is available. We compute the centroids for the question and the comment, then use the cosine between the two as a feature. The intention is to capture the similarity between different terms in the pair. The same procedure is then applied once more for only NP-S, i.e. noun phrase, tokens because they carry more information about the topic than other parts of speech.

\subsection{Classifier Model}

After all described features are extracted, they form a list of string values associated with each questionanswer pair. As explained above, some of them are characteristic for bad answers, while others are mainly found in good ones. Therefore, it makes sense to consider the feature list for a given questionanswer pair as a document itself. Classifying these documents with any standard approach will then group pairs with similar features together and will differentiate good from bad answers.

In our system, we use MALLET (McCallum and Kachites, 2002) to perform classification on the extracted feature documents. For classification we have chosen the default MALLET workflow that calculates term-frequency feature vectors from its input documents. These vectors are then fed to a MaxEnt classifier, trained and evaluated using tenfold cross validation. For the final classification, the trained classifier outputs class probabilities for each of the three desired categories: Good, Potential or Bad (which also includes Not English/Dialogue), and the one with the highest score is chosen as the label for the question-answer pair.

\section{Experiments and results}

Various experiments were conducted to analyse the contribution of the chosen features. In each of them, training was performed on the combined data from the train and development datasets, provided by the organizers. Testing was done on the official test dataset used for evaluation of the task, after it was released by the organizers. The analysis will only focus on the coarse-grained evaluation in the three main classes (Good, Potential, Bad) since our system does not try to target the finer-grained classification.

We defined our baseline system as the one that uses only the lexical and structural features described in the Method section, i.e. word tokens, sentence, question and answer length, as well as the bigrams and trigrams of the question-answer pair. With only these features, the system is very weak the accuracy as reported by the scorer script against the gold standard is $44.18 \%$ and the F1 score is $24.05 \%$.

Next, we included the features that rely on GATE gazetteers, such as the named entities features. This improved the system's performance by more than $1 \%$, reaching accuracy of $45.14 \%$ and $\mathrm{F} 1$ score of $25.33 \%$.

Another experiment we did was to add to the baseline system only the DKPro cosine similarity. This approach yielded a significant increase in the scores on the test set over the baseline system, around $4 \%$.

Finally, we tested the baseline system with the word2vec cosine values. This experiment was not as successful as the others, offering no improvement. The result may be attributed to the fact that we use a set of vectors trained on generic Web data instead of vectors specifically trained for the SemEval task. However, the community generated datasets are not sufficiently large and cannot be used for adequate word2vec training.

When all features were combined, the scores were boosted to $50 \%$ accuracy and $32.02 \% \mathrm{~F} 1$. The improvement from the baseline system is greater than the accumulated improvement from adding the single features because those features influence each other.

All of the described experiments were done on the data from the train and development sets. However, when preparing our final submission for the competition, we trained our system on a training set that included the development data twice. This way more weight was given to those question-answer pairs. The result was an impressive $14 \%$ increase in our F1 score.

In order to further analyse this surprising result, we did train a MaxEnt classifier using only the 
smaller development dataset. All described features were combined here as well. The experiment showed that indeed the larger train dataset provided for the competition has less effect on the performance of our system than the smaller development dataset. We suspect that the contents of the test dataset are closer to the development dataset because that would mean more common n-gram features are detected. This would explain the boost in the F1 score and the accuracy.

A summary of the results obtained in the experiments can be seen in Tables 1 and 2

\begin{tabular}{|l|c|c|}
\hline & Accuracy & F1 score \\
\hline baseline & $44.18 \%$ & $24.05 \%$ \\
\hline + gazetteers & $45.14 \%$ & $25.33 \%$ \\
\hline + cosine similarity & $47.87 \%$ & $28.98 \%$ \\
\hline + word2vec & $44.13 \%$ & $24.03 \%$ \\
\hline all combined & $50.00 \%$ & $32.02 \%$ \\
\hline \hline final system & $62.35 \%$ & $46.07 \%$ \\
\hline
\end{tabular}

Table 1: Accuracy and F1 score achieved using various combinations of features

\begin{tabular}{|l|c|c|}
\hline Training Data & Accuracy & F1 score \\
\hline Train + Devel & $50.00 \%$ & $32.02 \%$ \\
\hline Devel Only & $57.74 \%$ & $44.37 \%$ \\
\hline $\begin{array}{l}\text { Final System } \\
\text { (Train + 2* Devel) }\end{array}$ & $62.35 \%$ & $46.07 \%$ \\
\hline
\end{tabular}

Table 2: Accuracy and F1 score achieved using all features, but extracted from different training datasets

It should be noted that the results are greatly impacted by the low score we get on the Potential answers class. The scores on this label are very close to 0 with all devised systems, which is to be expected since none of our features were specifically targeted at distinguishing Potential answers from Good and Bad ones.

In all experiments, the highest precision and recall were achieved on the Bad answers.

\section{Conclusion}

In this paper we introduced our system for answer classification of question answering data. We described the method of preprocessing and applying features to the tokens and also mentioned the intergrated systems used for its implementation. All the steps of the data preparation for analysis were exhaustively described in the method description. Lexical and structural features proved to be insufficient for achieving high results. The gazetteers helped increase our scores but the most important part were the vector calculations made after the preparation process. The experiments showed that examining cosine distance between question and answer can lead to much greater performance. However, the most dramatic improvement was caused by increasing the size of the training data set and giving more weight to some question-answer pairs. For future work, we would try to add more syntactic features into the preprocessing and to integrate language models for the Good and Bad comments classification. With this system, we achieved satisfactory results for the SemEval 2015 answer-validation task.

\section{References}

McCallum, Andrew Kachites. 2002. "MALLET: A Machine Learning for Language Toolkit.” http://mallet.cs.umass.edu.

H. Cunningham, V. Tablan, A. Roberts, K. Bontcheva. 2013. Getting More Out of Biomedical Documents with GATE's Full Lifecycle Open Source Text Analytics. PLoS Comput Biol 9(2): e1002854. doi:10.1371/journal.pcbi.1002854 http://tinyurl.com/gate-life-sci/

H. Cunningham, et al. 2011. Text Processing with GATE (Version 6). University of Sheffield Department of Computer Science. 15 April 2011. ISBN 0956599311.

Tomas Mikolov, Ilya Sutskever, Kai Chen, Greg Corrado, and Jeffrey Dean. 2013. Distributed Representations of Words and Phrases and their Compositionality. In Proceedings of NIPS, 2013.

Tomas Mikolov, Kai Chen, Greg Corrado, and Jeffrey Dean. 2013. Efficient Estimation of Word Representations in Vector Space. In Proceedings of Workshop at ICLR, 2013.

Tomas, Wen-tau Yih, and Geoffrey Zweig. 2013. Linguistic Regularities in Continuous Space Word Representations. In Proceedings of NAACL HLT, 2013.

Daniel Bär, Torsten Zesch, and Iryna Gurevych. 2013. DKPro Similarity: An Open Source Framework for Text Similarity. In Proceedings of the 51st Annual Meeting of the Association for Computational Linguistics: System Demonstrations, pages 121-126, August 2013, Sofia, Bulgaria. 
Eckart de Castilho, R. and Gurevych, I. 2014. A broadcoverage collection of portable NLP components for building shareable analysis pipelines. In Proceedings of the Workshop on Open Infrastructures and Analysis Frameworks for HLT (OIAF4HLT) at COLING 2014, Dublin, Ireland.

Osman Başkaya. 2014. AI-KU: Using Co-Occurrence Modeling for Semantic Similarity. Artificial Intelligence Laboratory, Koç University, Istanbul, Turkey. SemEval-2014.

Darnes Vilarin̋o, David Pinto, Saúl León, Mireya Tovar, Beatriz Beltrán. 2014. BUAP: Evaluating Features for Multilingual and Cross-Level Semantic Textual Similarity. Benemérita Universidad Autónoma de Puebla Faculty of Computer Science, Puebla, México. SemEval-2014.

Magdalena Kacmajor, John D. Kelleher. 2014. DIT: Summarisation and Semantic Expansion in Evaluating Semantic Similarity. IBM Technology Campus, Applied Intelligence Research Centre, Dublin, Ireland. SemEval-2014. 